\title{
The Personality Differentiation between Dongxiang and Tibetan College Student
}

\author{
Lingkai Ma1,2,3, Congcong Ma4, Yaodong Jin 1,2,3 \\ ${ }^{1}$ Institute of Psychological Sciences, College of Education, Hangzhou Normal University, Hangzhou, China \\ ${ }^{2}$ Zhejiang Key Laboratory for Research in Assessment of Cognitive Impairments, Hangzhou, China \\ ${ }^{3}$ Center Cognition and Brain Disorder, Hangzhou Normal University, Hangzhou, China \\ ${ }^{4}$ Mental Health Education, College of Education, Hangzhou Normal University, Hangzhou, China \\ Email: 592970734@qq.com
}

How to cite this paper: Ma, L.K., Ma, C.C. and Jin, Y.D. (2020) The Personality Differentiation between Dongxiang and Tibetan College Student. Open Access Library Journal, 7: e6413.

https://doi.org/10.4236/oalib.1106413

Received: May 11, 2020

Accepted: June 1, 2020

Published: June 4, 2020

Copyright $\odot 2020$ by author(s) and Open Access Library Inc.

This work is licensed under the Creative Commons Attribution International License (CC BY 4.0).

http://creativecommons.org/licenses/by/4.0/

\begin{abstract}
Dongxiang and Tibetan college students can understand the personality characteristics, for the national colleges and universities students have a better communication and management of the theoretical data support. Selecting some proposals of Lanzhou city public universities, Dongxiang and Tibetan college students as subjects, using Roger, Chinese Adjectives Scale of Big-Five Factor Personality (referred to as "Big Five Personality Scale") compiled by Dai Xiaoyang, analyzed the personality differences of college students across the country and discussed its essence. Through the study, on the whole, the students of the two nationalities differ significantly in the seriousness of personality, and the college students of Dongxiang are significantly higher than that of Tibetan ethnic minority. In terms of gender, there are significant differences between the Dongxiang and the Tibetan female college students in the appropriate human dimension. There are not significant differences in the five personality dimensions of male college students. In terms of grades, freshmen and juniors in Dongxiang are significantly different from Tibetan students of the same grade. The freshmen of Dongxiang are significantly higher than the Tibetan freshmen in the extroversion dimension. There were significant differences in extroversion and conscientiousness among the Dongxiang and Tibetan students in rural (or pastoral) areas.
\end{abstract}

\section{Subject Areas}

Psychology, Personality

\section{Keywords}

Personality, Dongxiang, Tibetan 


\section{Introduction}

Personality is also called personality. Psychologists have different definitions of personality due to the different research directions of personality, but people have a certain degree of consensus on the basic characteristics of personality. Generally thinking that individual personality on the behavior of internal tendency, its performance for individual in ability to adapt to the environment, emotional needs, motivation, interests, attitudes, values, temperament, character and physical aspects of integration is a dynamic consistency and continuity of self, is formed in the course of socialization of individual psychosomatic group that gives a person with characteristics [1].

Dongxiang is a unique ethnic group that has lived in Gansu for generations. They originate from a branch of Mongolian and believe in Islam. Their way of life is a traditional production mode of male plowing and female weaving, which is an integral part of farming civilization [2]. Tibetans are traditional nomads, living a nomadic life. The two ethnic groups have their own cultural inheritance system and the thinking modes of different personality, manner and personality traits have bigger difference, for example, in the daily activities, Dongxiang taboo to mention pork and related things, etc., Tibetan taboo deception, etc., these are all have a certain relationship with the nation's personality characteristics [3] [4] [5] [6].

The development of personality affects the psychological development of a nation. Only by understanding the personality characteristics of this nation and making a comparison, can one play a positive role in the communication in the future. As a special minority in Gansu, Dongxiang believes in Islam and is influenced by family and religion since childhood, which makes their way of thinking very different from other ethnic groups. Another representative Tibetan, whose ancestors came from the Tibetan plateau, believed in Tibetan Buddhism, and some of them came to Gansu after historical migration. The two peoples are theoretically very different in interpersonal and other ways.

\section{The Present Investigation and Research Hypotheses}

\subsection{Literature Review}

Academia's research on personality differences between Dongxiang and Tibetan college students has not directly shown, and only some materials can be provided on the side. There are many researches on personality. For example, Huang Xiting's "Personality Psychology" published in 2002, and Jiang Zhi's "Western Psychologist's Theory of Personality Development Stage Theories" published in 1992 detailed the definition and role of personality set forth. Regarding the research on the personality of Dongxiang people, it is difficult to find documents that directly explain their personality. Yao Zhibin's "Clan, Belief and Construction of Ethnic Identity Construction" published in 2017 searched for Dongxiang people's personality. Academia's research on Tibetan personality involves various fields. Sang Jieduanzhi's “Tibetan Traditional Culture and Ti- 
betan Personality" published by Qinghai Social Sciences, and "The Status Quo of the Development of Tibetan College Students' Personalities and Their National Models" "Comparative Research" has made outstanding research on the personality characteristics of Tibetan college students. These studies have described and related the personality characteristics of Dongxiang and Tibetan college students [7].

\subsection{Research Hypotheses}

Some proposals based on this, this study in Gansu province and the Tibetan college students as research subjects, differences in personality assessment, Roger, Dai Xiao-yang compiled by Chinese adjective big five personality inventory (short form), the extraversion, neuroticism, conscientiousness, openness and agreeableness is evaluated, the five dimensions of personality differences as a result, the two studies on personality characteristics of college students, hope to conclude some research results and information. So that Dongxiang and Tibetan college students can find their own advantages and disadvantages, improve and develop themselves, can better shape their personality [8] [9]. According to the results of the personality traits tested, we can also plan a suitable plan and development direction for ourselves. The research on the personality characteristics of students has a great practical effect. We propose the following hypothesis:

Hypothesis 1: The overall personality characteristics of Dongxiang and Tibetan university students are significantly different.

Hypothesis 2: There are significant differences between Dongxiang and Tibetan college students in demographic variables.

Hypothesis 3: There are differences between Dongxiang and Tibetan college students in the five personality dimensions of extraversion, agreeableness, conscientio.

We combine the results of this study with previous literature to verify our hypothesis.

\section{Methodology}

\subsection{The Participants}

The object of the study participants was selected for study in Lanzhou city public university in Dongxiang and Tibetan college students, the principle of random sampling to choose the participants, some proposal as a result of living in Gansu province and the Tibetan people is limited, and the conditions of the participants limited to college students in Lanzhou city public universities, the final data statistical information, the effective questionnaire 334 . The background information of the participants is shown in Table 1.

\subsection{Research Tools}

The personality difference assessment scale of Dongxiang nationality and Tibetan nationality college students adopted the Chinese adjective big five personality scale 
Table 1. The background information of the participants.

\begin{tabular}{|c|c|c|c|}
\hline Variable & & Population & Portion \\
\hline \multirow[t]{2}{*}{ National } & Dongxiang & 165 & $49.4 \%$ \\
\hline & Tibetan & 169 & $50.6 \%$ \\
\hline \multirow[t]{2}{*}{ Gender } & Male & 173 & $51.8 \%$ \\
\hline & Female & 161 & $48.2 \%$ \\
\hline \multirow[t]{4}{*}{ Grade } & Freshman & 86 & $25.7 \%$ \\
\hline & Sophomore year & 36 & $10.8 \%$ \\
\hline & Junior & 93 & $27.8 \%$ \\
\hline & Senior & 119 & $35.7 \%$ \\
\hline \multirow[t]{2}{*}{ Origin of student } & Town & 75 & $22.5 \%$ \\
\hline & $\begin{array}{l}\text { Countryside } \\
\text { (Pastoral) }\end{array}$ & 59 & $77.5 \%$ \\
\hline \multirow[t]{2}{*}{ Discipline } & Science & 201 & $60.2 \%$ \\
\hline & Arts & 133 & $39.8 \%$ \\
\hline
\end{tabular}

(simplified version) compiled by Roger and Dai Xiaoyang.

The questionnaire format adopted the Chinese format for the Dongxiang nationality whose mother tongue was Chinese, and the Tibetan-Chinese bilingual format for the Tibetan nationality whose mother tongue was Tibetan, which was helpful to enhance the subjects' sense of identity to the questionnaire and improve the quality and recovery of the questionnaire. The big five personality model includes five personality dimensions of extraversion, neuroticism, conscientiousness, openness and agreeableness, and contains 20 items. The internal consistency (coefficient) is $0.85,0.72,0.72,0.78$ and 0.85 , respectively. The $\mathrm{P}$ values are all less than 0.01 , which has a high reliability index.

The test items are in the form of bipolar adjectives, and the scoring method is a six-point scoring method. Take "extrovert-introvert" as an example, $1=$ fully introverted, $2=$ more extroverted, $3=$ somewhat extroverted, $4=$ somewhat introverted, 5 = somewhat introverted, 6 = fully introverted. Scoring method: first, reverse scoring is carried out on the reverse questions, and then adds up the questions of each dimension to get the score of each dimension [10].

\subsection{Procedure}

In this study, the method of random sampling was selected. After obtaining the consent of the subjects, the instructions were read to the subjects, and the subjects were asked to read them in detail. After the subjects got familiar with the instructions, the subjects were selected according to the actual situation. After the respondents answered the questionnaire, they immediately collected it, collected the questionnaire for statistics and processing, and then screened out the invalid questionnaire, so as to ensure scientific and orderly processing of the questionnaire. 


\subsection{Data Analysis}

After the questionnaire was collected, the data were sorted out and checked, the quantity and quality of the questionnaire were counted again, and the correctness of the data was determined. The sorted questionnaires were coded and recorded to ensure that all the questionnaires could be recorded into the system. SPSS19 was used for statistical processing and data analysis of the sorted data.

\section{Results}

\subsection{Analysis of the Overall Personality Difference between Dongxiang and Tibetan University Students}

The independent sample $t$ test was used to test the differences in personality of college students of different nationalities. The results are shown in Table 2.

It can be seen from Table 2 that the conscientiousness dimension of college students' personality is significantly different among different ethnic groups. College students of Dongxiang are significantly higher than those of Tibetan, and there is no significant difference in other dimensions of personality, $t$ is 1.97, there is a significant difference between the two.

\subsection{The Personality Differences in College Student between Dongxiang and Tibetan}

The personality differences in first-year students between Dongxiang and Tibetan. The independent sample t-test showed that the Dongxiang and Tibetan first-year student had diverse personality. Specifically, the students of Dongxiang had higher Extroversion and Agreeableness than the Tibetan students (Table 3).

The personality differences between Dongxiang and Tibetan Sophomores. The independent sample t-test failed to find the significant personality differences between Dongxiang and Tibetan sophomores. Specifically, the Dongxiang and Tibetan sophomores showed the same personality of extroversion $(t=0.09$, $\mathrm{p}>0.05)$, agreeableness $(\mathrm{t}=1.09, \mathrm{p}>0.05)$, conscientiousness $(\mathrm{t}=1.44, \mathrm{p}>$ $0.05)$, neuroticism $(t=-0.49, \mathrm{p}>0.05)$, openness $(\mathrm{t}=-0.81, \mathrm{p}>0.05)$.

The personality differences of the junior between Dongxiang and Tibetan. The independent sample t-test showed that the junior of Dongxiang and Tibetan had

Table 2. Personality differences between Dongxiang and Tibetan female college students.

\begin{tabular}{cccc}
\hline Dimension & $\begin{array}{c}\text { Dongxiang } \\
\mathbf{M} \pm \text { SD }\end{array}$ & $\begin{array}{c}\text { Tibetan } \\
\mathbf{M} \pm \text { SD }\end{array}$ & $\boldsymbol{t}$ \\
\hline Extraversion & $15.87 \pm 4.46$ & $14.98 \pm 4.19$ & 1.87 \\
Agreeableness & $16.81 \pm 4.43$ & $16.24 \pm 3.68$ & $1.97^{*}$ \\
Conscientiousness & $15.54 \pm 3.86$ & $14.76 \pm 3.30$ & -0.35 \\
Neuroticism & $12.72 \pm 3.98$ & $12.86 \pm 3.44$ & 0.63 \\
Openness & $15.02 \pm 04.08$ & $14.75 \pm 3.70$ & \\
\hline
\end{tabular}

Note: ${ }^{*}$ indicates $\mathrm{p}<0.05$. 
Table 3. The personality differences of first-year students between Dongxiang and Tibetan.

\begin{tabular}{cccc}
\hline Dimension & $\begin{array}{c}\text { Dongxiang } \\
\mathbf{M} \pm \mathbf{S D}\end{array}$ & $\begin{array}{c}\text { Tibetan } \\
\mathbf{M} \pm \mathbf{S D}\end{array}$ & $\mathbf{t}$ \\
\hline Extroversion & $16.91 \pm 4.31$ & $14.67 \pm 4.83$ & $2.26^{\star}$ \\
Agreeableness & $18.26 \pm 3.75$ & $16.02 \pm 3.00$ & $2.97^{\star}$ \\
Conscientiousness & $16.18 \pm 3.58$ & $15.05 \pm 3.28$ & 1.49 \\
Neuroticism & $11.14 \pm 3.20$ & $12.59 \pm 3.87$ & -1.89 \\
Openness & $16.12 \pm 3.95$ & $15.13 \pm 4.25$ & 1.11 \\
\hline
\end{tabular}

Note: ${ }^{*}$ means $\mathrm{p}<0.05$.

Table 4. The personality differences of the junior between Dongxiang and Tibetan.

\begin{tabular}{cccc}
\hline Dimension & $\begin{array}{c}\text { Dongxiang } \\
\mathbf{M} \pm \mathrm{SD}\end{array}$ & $\begin{array}{c}\text { Tibetan } \\
\mathbf{M} \pm \mathrm{SD}\end{array}$ & $\mathbf{t}$ \\
\hline Extroversion & $16.78 \pm 4.17$ & $14.76 \pm 4.24$ & $2.25^{*}$ \\
Agreeableness & $16.75 \pm 4.19$ & $16.28 \pm 4.31$ & 0.52 \\
Conscientiousness & $15.18 \pm 3.02$ & $14.26 \pm 3.59$ & 1.28 \\
Neuroticism & $12.83 \pm 3.49$ & $13.26 \pm 3.40$ & -0.58 \\
Openness & $15.24 \pm 3.72$ & $14.57 \pm 3.61$ & 0.86 \\
\hline
\end{tabular}

differences in their personality. The extroversion of Dongxiang student was significantly higher than the Tibetan student $(\mathrm{t}=2.26, \mathrm{p}<0.05)$.

The personality differences of the senior between Dongxiang and Tibetan. The independent sample t-test failed to find the significant personality differences between Dongxiang and Tibetan senior student. Specifically, the senior of Dongxiang and Tibetan showed the same personality of extroversion $(t=-0.82$, $\mathrm{p}>0.05)$, agreeableness $(\mathrm{t}=-1.23, \mathrm{p}>0.05)$, conscientiousness $(\mathrm{t}=-0.17, \mathrm{p}>$ $0.05)$, neuroticism $(t=1.96, p>0.05)$, openness $(t=0.83, p>0.05)$.

\subsection{The Personality Differences between Dongxiang and Tibetan College Students from Different Region}

The personality differences between Dongxiang and Tibetan college students who is from urban. The independent sample $t$-test failed to find the significant personality differences between Dongxiang and Tibetan student who is from urban. Specifically, the Dongxiang and Tibetan students showed the same personality of extroversion $(t=0.36, p>0.05)$, agreeableness $(t=-0.45, p>0.05)$, conscientiousness $(t=0.16, p>0.05)$, neuroticism $(t=0.18, p>0.05)$, openness $(\mathrm{t}=-0.71, \mathrm{p}>0.05)$.

The personality differences between Dongxiang and Tibetan college students in rural areas (or pastoral areas). The independent sample t-test showed that the Dongxiang and Tibetan students who came from the rural areas or pastoral areas had differences in their personality. The Dongxiang student has higher extroversion and conscientiousness than the Tibetan student $(\mathrm{t}=1.98, \mathrm{p}<0.05 ; \mathrm{t}=2.25$, $\mathrm{p}<0.05)$ (Table 4, Table 5). 
Table 5. The personality differences between Dongxiang and Tibetan college students in rural areas (or Pastoral Areas).

\begin{tabular}{cccc}
\hline Dimension & $\begin{array}{c}\text { Dongxiang } \\
\mathbf{M} \pm \mathrm{SD}\end{array}$ & $\begin{array}{c}\text { Tibetan } \\
\mathbf{M} \pm \mathrm{SD}\end{array}$ & $\mathbf{t}$ \\
\hline Extroversion & $15.79 \pm 4.11$ & $14.79 \pm 3.95$ & $1.98^{*}$ \\
Agreeableness & $16.92 \pm 4.17$ & $16.05 \pm 3.51$ & 1.81 \\
Conscientiousness & $15.68 \pm 3.66$ & $14.71 \pm 3.24$ & $2.25^{\star}$ \\
Neuroticism & $12.66 \pm 3.72$ & $12.90 \pm 3.29$ & -0.55 \\
Openness & $15.02 \pm 3.80$ & $14.48 \pm 3.57$ & 1.18 \\
\hline
\end{tabular}

Note : ${ }^{\star}$ means $\mathrm{p}<0.05$.

\subsection{Personality Differences between Dongxiang and Tibetan College Students in the Same Discipline}

Personality Differences between Dongxiang and Tibetan college students who majored in liberal arts and social science. The independent sample t-test failed to find the significant personality differences between Dongxiang and Tibetan student who studied liberal arts. Specifically, the Dongxiang and Tibetan students showed the same personality of extroversion $(t=0.99, p>0.05)$, agreeableness ( $t$ $=0.38, \mathrm{p}>0.05)$, conscientiousness $(\mathrm{t}=1.46, \mathrm{p}>0.05)$, neuroticism $(\mathrm{t}=-0.19$, $\mathrm{p}>0.05)$, openness $(\mathrm{t}=1.34, \mathrm{p}>0.05)$.

Personality Differences between Dongxiang and Tibetan college students who majored in STEM (Sciences, Technology, engineering and mathematics). The independent sample t-test failed to find the significant personality differences between Dongxiang and Tibetan student who studied liberal arts. Specifically, the Dongxiang and Tibetan students showed the same personality of extroversion $(t=1.81, p>0.05)$, agreeableness $(t=1.60, p>0.05)$, conscientiousness $(t=$ $1.30, \mathrm{p}>0.05)$, neuroticism $(\mathrm{t}=-0.42, \mathrm{p}>0.05)$, openness $(\mathrm{t}=-0.44, \mathrm{p}>0.05)$.

\section{Discussion}

\subsection{The Differences between Dongxiang and Tibetan College Students in Big Five Personality Traits}

According to the independent sample $\mathrm{t}$ test, the Dongxiang students had higher conscientiousness and extroversion than the Tibetan students, but there were no significant differences between Dongxiang and Tibetan students in the other three personality traits. This may due to the reason that the Tibetans are nomads. They used to live in tents and migrate frequently. These lifestyles make them more independent and casual. As for the Dongxiang people, they are concentrated in the plains, mainly farming or doing business. These activities are highly dependent on the order and have strong demand for interpersonal communication. Engaging in these activities for a long time helps the Dongxiang people form the characteristics of outgoing, orderliness, and self-discipline, which makes them more conscientiousness and extroversion than the Tibetan people [11]-[16]. 
Despite these differences, the Dongxiang people and the Tibetan have much in common in the other aspects. For instance, both Dongxiang and Tibetan people have religious beliefs. Although the Dongxiang people believe in Islam and the Tibetans believe in Tibetan Buddhism, these two religious both emphasize some universal values such as kindness, peaceful. Dongxiang and Tibetans have believed in their religion for generations and these religions taught them how to behave and how get along with others. Therefore, the Dongxiang and Tibetans students have some common personality traits, such as agreeableness.

\subsection{The Personality Differences between Dongxiang and Tibetan College Students for the Same Gender}

There is a significant difference in the personality of agreeableness between the female Dongxiang and Tibetan college students, but they not differed in the other four personality. For the male Dongxiang and Tibetan college students, they showed the same personality in both five dimensions.

The above phenomenon may due to the reason that both Dongxiang and Tibetans live in the western region, and they have similar views on the status of men and women. In their culture, men are the pillars of a home, they have power to make the decision about both big things and small things. Therefore, the male Dongxiang students and Tibetan students showed the same personality. As for the women, Tibetan women is not good at socializing, they rarely married to other ethnic groups and think only man of their own ethnic group are worthy of reliance. Therefore, they are shy and cautious than Tibetan women. That is why the female Dongxiang students are more agreeableness than the Tibetan female students.

\subsection{Personality Differences between Dongxiang and Tibetan College Students of the Same Grade}

Regarding the differences in grades, Dongxiang college students in grade one are significantly more extroverted than Tibetan students, which is consistent with the research hypothesis. Because most Dongxiang college students come from areas where many ethnic groups live together, they have experienced many other ethnic groups before entering the university. They live and grow together. For freshmen entering university, they just changed their living environment. They are full of novelty and interest in everything at the university, and they are more adept at dealing with others; Tibetan students generally come from the Tibetan area, and most of them have been in contact with Tibetans since childhood. They lack communication with the outside world and with other ethnic groups. Compared with Dongxiang college students, their inside world is relatively conservative, and they are not good at communicating with the outside world. The habits and habits they have developed from a young age make them have a big gap with the outside world. It is said that they have not really integrated into this large environment. Although they are full of curiosity about the new environment, they are closed to themselves due to rigor and kindness. This is why, 
among freshmen, Dongxiang students are significantly higher than Tibetan college students in both extroversion and agreeableness. Sophomores have fully adapted to the university's living conditions and lifestyles after one year of study and life, and the impact they have made them have similarities in the five dimensions of personality. In the third grade, Dongxiang students are significantly more extroverted than Tibetan students. This is influenced by personal thoughts, because the senior year is about to face a period of graduation to find a job, start an internship, and determine the employment direction. Tibetan college students are more inclined returning to the land where they grew up, they want to go back to Tibet, so they have no intention of staying in a foreign city that is not their hometown. Dongxiang college students are more inclined to find jobs suitable for their own development prospects, and often need to participate in recruitment and interview and other activities, it is good at active and diplomatic, so in the third grade, Dongxiang college students are significantly more extroverted than Tibetan students [17] [18].

\subsection{Personality Differences between Dongxiang and Tibetan College Students in the Same Birthplace}

The rural (or pastoral) college students of the Dongxiang and Tibetan ethnic groups have significant differences in personality and extroverted dimensions. Among them, the rural (or pastoral) college students of the Dongxiang and Tibetan ethnic groups are significantly higher than the Tibetan rural (or pastoral) dimensions. College students, in terms of conscientiousness dimensions, Dongxiang rural (or pastoral) college students are significantly higher than Tibetan rural college students; there is no significant difference in personality between Dongxiang and Tibetan college students from urban areas. This is consistent with research assumptions. Although Dongxiang and Tibetan college students from cities and towns believe in different religions, the life pattern and lifestyle of living in cities and towns are the same. The education and ideas they receive from childhood are the same. The thinking they contacted is also advanced, and the influence of family and friends around are the same, which makes them roughly the same in these five dimensions of personality, and there is not much difference. The reason for this difference can be based on their living environment. Tibetans live in plateau regions with high and cold climates. They are nomads. Most of the Tibetans living in rural areas (or pastoral areas) live by water and grass. Dongxiang Living in the plains, Dongxiang people from rural (or pastoral) families depend on farming for their livelihood. Different production methods also make them different. This also shows that, in terms of the openness dimension, Dongxiang rural (or pastoral) college students are significantly higher than Tibetan rural (or pastoral) college students; in terms of conscientiousness dimension, Dongxiang rural (or pastoral) college students are significantly higher than Tibetan rural College students; and the conclusion is that there is no significant difference in personality between Dongxiang and Tibetan college students from urban areas. 


\subsection{Personality Differences between Dongxiang and Tibetan College Students in the Same Discipline}

There is no significant difference in personality dimension between college students of different disciplines of Dongxiang and Tibetan, which is in consisted with research assumptions. Individual's character develops slowly from an early age, and is formed through the combination of internal and external influences. During the schooling period, Dongxiang and Tibetan college students studied together after receiving compulsory education and received the same education. They also cultivated their problem thinking and problem-solving abilities and conducted scientific planning teaching. Each student accepts the same education and development here, so there is no dissertation or science and engineering. Because the educational concepts and methods of education are the same, there is no difference between Dongxiang and Tibetan college students in various personality dimensions [19].

\section{Conclusions}

\subsection{Different Degrees of Personality Differences between Dongxiang and Tibetan College Students}

The rigorous dimension of the personality of college students differs significantly among different ethnic groups. Dongxiang college students are significantly higher than Tibetan college students. The other dimensions of personality do not differ significantly among different ethnic groups.

\subsection{There are Different Degrees of Personality Differences in Gender between Dongxiang and Tibetan College Students}

In terms of gender, Dongxiang female college students are significantly more agreeableness than Tibetan female college students; Dongxiang and Tibetan male college students have no significant differences in the five personality dimensions.

\subsection{There are Different Degrees of Personality Differences between Dongxiang and Tibetan College Students in Grades}

In terms of grades, in the freshman year, Dongxiang college students are significantly higher than Tibetan college students in two dimensions of extroversion and agreeableness. In the third grade, Dongxiang college students are significantly more agreeable than Tibetan college students.

\subsection{Different Degrees of Personality Differences in the Birthplace of Dongxiang and Tibetan College Students}

In terms of birthplace, there are significant differences in personality and extroversion between Dongxiang and Tibetan rural (or pastoral) college students; there is no significant difference in personality between Dongxiang and Tibetan college students from urban areas. 


\subsection{There is no Personality Difference between the Disciplines of Dongxiang and Tibetan College Students}

There is no significant difference in personality dimension between Dongxiang and Tibetan college students in different disciplines.

\section{Summary and Outlook}

In terms of the participants, in this study, because the selected subjects were Dongxiang and Tibetan college students living in Gansu, the sample size was small, and it was relatively complicated and difficult to find the participants. Random search, when introducing the subject by others, the selection of participants is more tortuous and inconvenient. For future research, multiple methods should be used to find participants, and a combination of multiple methods should be used to issue questionnaires to find participants.

Moreover, the scope of the selected participants is too limited. Only Dongxiang and Tibetan undergraduates who are studying in Lanzhou City are selected. This limits the research participants to a small range and lacks broadness. In the future, the scope of Dongxiang and Tibetan college students cannot be limited to Lanzhou, but can be found in other cities in Gansu Province and nearby provinces and cities in Gansu Province. Nationalities and young people with higher education, such as students and postgraduates, should expand the scope of their research objects.

The results of this study showed that the differences in the effects of the Big Five personality are not too obvious. The cause of the problem is not concentrated when the questionnaire is issued, and the distribution of Dongxiang and Tibetan college students is not concentrated. For future research, we must pay full attention to this issue. When selecting participants, we must use new ideas and new ideas to select participants.

The questionnaire used the Chinese Adjective Big Five Personality Scale (Simplified Version) compiled by Luo Jie and Dai Xiaoyang. The questionnaire's dimension setting of the questionnaire was not obvious, which led the participants to choose " 3 " " 4 ", some significant differences in the data obtained are not obvious. In future research, the choice of questionnaires should be based on the combination of theory and actual conditions [20].

For the conclusions and basis of this research, a large number of correct and reliable arguments and research data are still needed to support it, and it needs to be combined with objective and accurate literature reviews.

\section{Contribution and Significance}

Back to previous studies, few studies have compared the differences in personality between Dongxiang and Tibetan students, and even fewer have explored the influence of disciplinary and cultural differences on the differences in personality between two different ethnic groups. The study of personality differences among different nationalities is conducive to the promotion of ethnic integration 
and unity, as well as the understanding of personality differences among different nationalities, which provides a theoretical basis for the study of ethnic psychology.

This study provides a basis and method for the study of personality differences among different ethnic groups, and provides a driving force for the study of personality differences among different ethnic groups. It encourages researchers to expand the research scope in the subsequent studies and include different ethnic groups into their own research scope.

\section{Conflicts of Interest}

The authors declare no conflicts of interest regarding the publication of this paper.

\section{References}

[1] Huang, X.T. (2002) Personality Psychology. Zhejiang Education Press, Hangzhou.

[2] (1984) A Brief History of Dongxiang. Gansu Nationalities Press, Lanzhou.

[3] Wang, X.Q., Zhang, J.C. and Luo, L. (2007) A Comparative Study of the Personality Traits of Minority College Students. Abstracts of Papers of the 11th National Psychological Conference, Kaifeng.

[4] Xue, J. and Chen, J. (2015) A Study on the National Self-Positive Prejudice of College Students of Different Nationalities. Abstracts of Papers of the 18th National Psychological Conference, Tianjin.

[5] Tang, B.L. and Zhai, S.L. (1997) On the Personality and Psychological Characteristics of Brother College Students. Journal of Sichuan Teachers College (Philosophy and Social Sciences), 1, 116-120.

[6] Kang, Y.W., Kang, X.W., Xie, S.J., Li, P. and Chen, C. (2012) Personality Characteristics of Tibetan College Students in the Mainland. Chinese School Health, 10, 1176-1179.

[7] Wang, Z.H. and Zhang, J.X. (2007) Influence of Gender, Gender Role and Gender Concept on Personality Traits of College Students. Psychological Development and Education, 23, 50-55.

[8] Jia, W.H. (2012) The Relationship between Personality Traits, Coping Styles and Psychological Adaptation of Rural Left-behind Children. Psychological Science, 1, 142-147.

[9] Zhao, J.C. and Ma, X.Y. (2001) Study on Personality Traits of Poor Students. Journal of Mathematical Education, 10, 9-16.

[10] Sui, R. (2012) A Study on the Personality Traits of Freshmen in Ethnic Minority Universities: A Case Study of South-Central University for Nationalities. South-Central University for Nationalities, Wuhan.

[11] Liu, X.D. (2003) The descendants of the Beizhuang family-Dongxiang. Yunnan People's Publishing House, Kunming.

[12] Zhang, L.J. (2007) Research on Dongxiang Poverty and Anti-Poverty. National Press, Lanzhou.

[13] Ma, Y.P. and Wang, L. (2003) Review of Dongxiang Studies in the Past 20 Years. Journal of the Second Northwest University for Nationalities (Philosophy and Social Sciences Edition), 3, 21-24. 
[14] Yu, L. (2013) Investigation and Research on Dongxiang Culture from the Perspective of Sheep.

[15] Wang, N.N. (2012) Personality and Mental Health of Tibetan Adolescents. Tibet University, Lhasa.

[16] Ran, R., and Yang, Y.X. (2012) Personality Traits of Tibetan Junior Middle School Students in the Mainland. Chinese Journal of Health Psychology, 12, 23-28.

[17] Long, Z.Q. and Li, C. (2018) The Status Quo of Personality Development of Tibetan College Students and a Comparative Study with National Norms. Journal of Higher Correspondence Education (Philosophy and Social Sciences Edition), 9, 36-39.

[18] Luo, J., Zhou, Y., Chen, W., Pan, Y. and Zhao, S.Y. (2016) Reliability Generalization Analysis of Big Five Personality Test in China. Psychological Development and Education, 1, 121-128.

[19] Yao, R.S. (2019) Simplified version of the Big Five Personality Scale_NEO_FFI_ Application Analysis in the College Student Population.

[20] Sangjie, Z.D. (2006) Tibetan Traditional Culture and Tibetan Personality. Qinghai Social Sciences, 1, 117-122. 